\title{
Chaotic saddles at the onset of intermittent spatiotemporal chaos
}

\author{
Erico L. Rempel* \\ Institute of Aeronautical Technology (ITA) and World Institute for Space Environment Research (WISER), CTA/ITA/IEFM, \\ São José dos Campos, São Paulo 12228-900, Brazil \\ Abraham C.-L. Chian and Rodrigo A. Miranda ${ }^{\dagger}$ \\ National Institute for Space Research (INPE) and World Institute for Space Environment Research (WISER), P.O. Box 515, \\ São José dos Campos, São Paulo 12227-010, Brazil \\ (Received 9 August 2007; published 30 November 2007)
}

\begin{abstract}
In a recent study [Rempel and Chian, Phys. Rev. Lett. 98, 014101 (2007)], it has been shown that nonattracting chaotic sets (chaotic saddles) are responsible for intermittency in the regularized long-wave equation that undergoes a transition to spatiotemporal chaos (STC) via quasiperiodicity and temporal chaos. In the present paper, it is demonstrated that a similar mechanism is present in the damped Kuramoto-Sivashinsky equation. Prior to the onset of STC, a spatiotemporally chaotic saddle coexists with a spatially regular attractor. After the transition to STC, the chaotic saddle merges with the attractor, generating intermittent bursts of STC that dominate the post-transition dynamics.
\end{abstract}

DOI: 10.1103/PhysRevE.76.056217

PACS number(s): 05.45.Jn, 47.27.Cn

\section{INTRODUCTION}

Chaotic dynamics can appear in the form of asymptotic or transient chaos. In dissipative systems, asymptotic chaos refers to the dynamics on chaotic attractors, and it is a wellknown fact that transient chaos is caused by the presence of nonattracting chaotic sets known as chaotic saddles in the phase space [1-4]. Random initial conditions usually spend some time in the vicinity of a chaotic saddle before escaping toward an attractor. When a system displays transient chaos and multistability, i.e., coexistence of multiple attractors, a chaotic saddle lies in the fractal basin boundaries $[5,6]$.

If two or more chaotic saddles are embedded in a chaotic attractor, trajectories on the attractor can visit the neighborhood of each saddle, experiencing different chaotic transients. The recurrence of these transient states generates intermittency [7]. Intermittency is a striking feature in nonlinear systems and has attracted much attention from both the chaos and turbulence communities. Different types of intermittency have been reported in chaotic systems, such as Pomeau-Manneville intermittency (alternation between chaotic and periodic behavior) [8], crisis-induced intermittency (alternation between two different chaotic behaviors) [9], and spatiotemporal intermittency (space-time mixture of fluctuating spatially ordered domains and "turbulent" patches) $[10,11]$. In hydrodynamic turbulence, intermittency consists of episodic switching of regions of strong vorticity and regions of relatively quiet fluid flow $[12,13]$.

A series of works have been published on the role of chaotic saddles in intermittency modeled by partial differential equations in regimes of temporal chaos (TC) $[4,14,15]$ and spatiotemporal chaos (STC) [16]. In the former case, the system is temporally chaotic and spatially regular, whereas

\footnotetext{
*rempel@ita.br

${ }^{\dagger}$ Also at Centre for Quaternary Research (CEQua), P.O. Box 113-D, Punta Arenas, Chile.
}

the latter case consists of both temporal chaos and spatial disorder. In [16] chaotic saddles were shown to be responsible for a type of intermittency involving random switchings between periods of temporal and spatiotemporal chaos (TCSTC intermittency) in a nonlinear regularized long-wave model in a small spatial domain. The aim of this paper is to show that the same mechanism can be found in the damped Kuramoto-Sivashinsky (KS) equation, a widely studied reaction-diffusion equation. In a large spatial domain, it is shown that the spatial complexity and temporal chaoticity of the attractor in the STC regime are basically determined by a spatiotemporally chaotic saddle present in the phase space for all values of the control parameter used in this study. In particular, we investigate the variation of spatial dynamics before and after transition to intermittent spatiotemporal chaos.

In Sec. II the damped Kuramoto-Sivashinsky equation and its numerical solution are presented. Section III describes the transitions from a periodic to a quasiperiodic, then to a temporally chaotic, and finally to a spatiotemporally chaotic attractor. Section IV discusses the role of chaotic saddles in spatiotemporally chaotic transients and TCSTC intermittency. The conclusions are given in Sec. V, where we suggest that a crisis is responsible for the transition to STC.

\section{THE KURAMOTO-SIVASHINSKY EQUATION}

The Kuramoto-Sivashinsky equation was named after its derivation by Kuramoto and Tsuzuki [17] as a phase equation for the complex amplitude of the Ginzburg-Landau equation, and by Sivashinsky [18] as a model of hydrodynamical instability in laminar flame fronts. It had been previously derived to describe the nonlinear saturation of the collisional trapped-ion mode, a drift wave associated with the oscillation of plasma particles trapped in magnetic wells created by the inhomogeneous magnetic field of a tokamak, where periodic boundary conditions are specified $[19,20]$. 
The damped Kuramoto-Sivashinsky equation is given by $[10,21]$

$$
\partial_{t} u=\left[\nu-\left(1+\partial_{x x}\right)^{2}\right] u-u \partial_{x} u,
$$

where $\nu \in[0,1]$ is a damping parameter. The mathematical properties and nonlinear dynamics of the KS equation for $\nu=1$ have been extensively studied $[4,10,14,15,22-26]$. Most works focus on the transition from order to temporal chaos, where spatial regularity is maintained and the KS equation resembles a low-dimensional dynamical system. Transition to spatiotemporal chaos as a function of the damping parameter $\nu$ was studied by Chaté and Manneville [10] for rigid boundary conditions. They observed the presence of transient spatiotemporally disordered states for $\nu$ below a critical value $\nu_{c}$, where the system undergoes transition from lamellar to spatiotemporal chaos. For $\nu$ above $\nu_{c}$, intermittent spatiotemporal chaos is observed.

The present paper investigates the transition to spatiotemporal chaos in the damped $(\nu<1) \mathrm{KS}$ equation with periodic boundary conditions $u(x, t)=u(x+L, t)$. Following Elder et al. [21], we take $L=536$. The transition point to spatiotemporal chaos seems to be independent of the system size for large enough $L[21,27]$.

Equation (1) is solved using a standard forward-time, centered-space finite-difference code, where the first-order forward-difference approximation is used for the time derivative and the second-order centered difference is employed for the spatial derivatives. A spatial grid with $N=1024$ points is adopted $(\Delta x \approx \pi / 6)$, with a time step $\Delta t=0.01$. The numerical scheme is stable for this choice of spatial and time steps, and one can study the late-time dynamics of Eq. (1). In order to simplify visualization of the outputs, a Poincaré map is defined by $u(5 \Delta x, t)=3$ and $\partial_{t} u(5 \Delta x, t)>0$. This choice of map is arbitrary and is found after observing the flow of Eq. (1) in the phase space. Implementation of the Poincaré map is performed using the Hénon method [28].

\section{TRANSITION TO SPATIOTEMPORAL CHAOS}

By applying small increments to the control parameter $\nu$ while following a particular set of initial conditions, the evolution of attractors of Eq. (1) can be investigated. Figure 1 shows the contour plots for the spatiotemporal patterns at four values of the damping parameter $\nu$. The first three patterns [Figs. 1(a)-1(c)] represent spatially regular regimes, whereas the fourth one [Fig. 1(d)] represents a spatially irregular regime. The pattern in Fig. 1(a) represents the spatiotemporal evolution of a trajectory on a periodic attractor of Eq. (1) at $\nu=0.625$. In Fig. 1(b) the periodic attractor evolves into a quasiperiodic attractor at $\nu=0.632$; Fig. 1(c) displays a temporally chaotic attractor at $\nu=0.635$; and Fig. 1(d) displays a spatiotemporally chaotic attractor at $\nu=0.637$. The temporal dynamics can be characterized by the maximum Lyapunov exponent $\lambda_{\max }$, computed by the Bennetin method $[29,30]$. For periodic and quasiperiodic regimes, $\lambda_{\max }=0$, whereas at $\nu=0.635$ the attractor is weakly chaotic, with $\lambda_{\max } \approx 0.007$. At $\nu=0.637$ the Lyapunov exponent suddenly jumps to $\lambda_{\max } \approx 0.2$, indicating a transition to
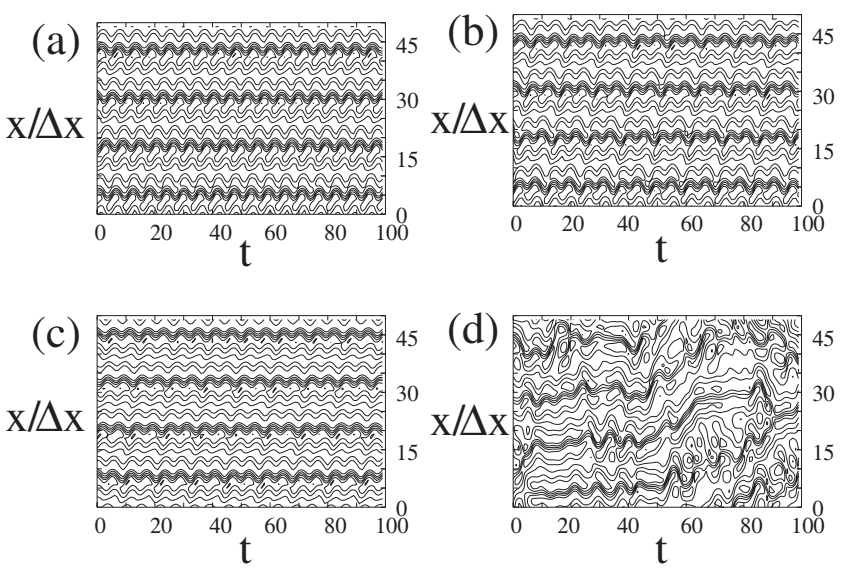

FIG. 1. Contour plots for the spatiotemporal patterns of Eq. (1) for (a) periodic attractor at $\nu=0.625$; (b) quasiperiodic attractor at $\nu=0.632$; (c) temporally chaotic attractor at $\nu=0.635$; (d) spatiotemporally chaotic attractor at $\nu=0.637$.

strong chaos within the STC regime, as can be seen in Fig. 2(a), which shows the convergence of $\lambda_{\max }$ for different sets (the Lyapunov exponent of the spatiotemporally chaotic saddle, STCS, in Fig. 2 is discussed in the next section).

The time-averaged power spectrum is a valuable tool to characterize the degree of spatial disorder. Figure 3 plots two time-averaged power spectra $\left\langle|\hat{u}|^{2}\right\rangle$, where $\hat{u}$ is the discrete spatial Fourier transform of $u(x, t)$ :
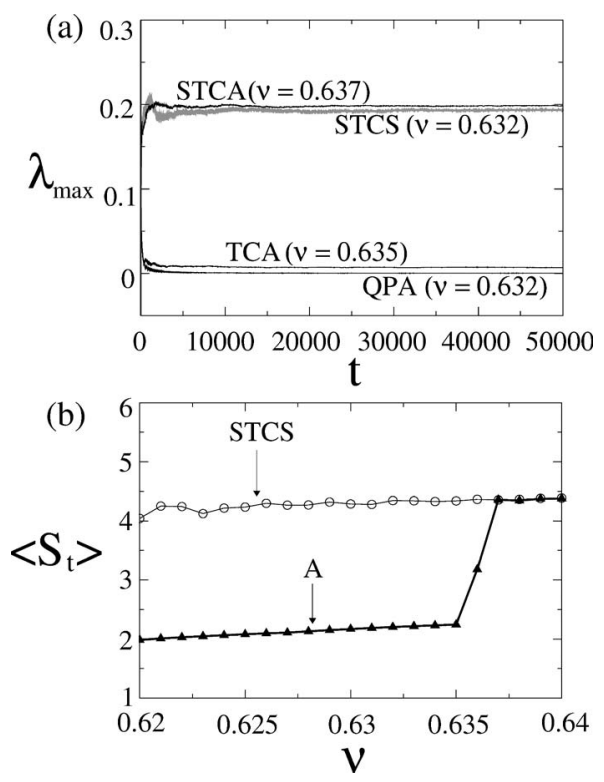

FIG. 2. (a) Maximum Lyapunov exponents $\lambda_{\max }$ for the attractors (black lines QPA, TCA, and STCA) and for the chaotic saddle (gray line, STCS) for different values of $\nu$; (b) variation of the time-averaged spectral entropy $\langle S\rangle_{t}$ with $\nu$ for the attracting sets (A, triangles) and for the spatiotemporally chaotic saddle (STCS, circles). 

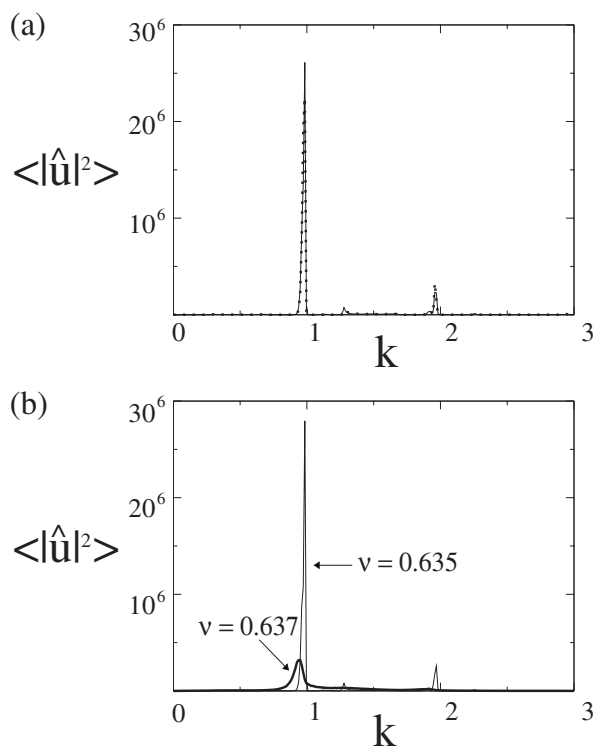

FIG. 3. Time-averaged power spectra for (a) periodic attractor $(\nu=0.625$, dotted line $)$ and quasiperiodic attractor $(\nu=0.632$, solid line); (b) temporally chaotic attractor $(\nu=0.635$, light line $)$ and spatiotemporally chaotic attractor $(\nu=0.637$, dark line $)$.

$$
\hat{u}(k, t)=\sum_{j=0}^{N-1} u(j \Delta x, t) e^{-\sqrt{-1} j \Delta x k},
$$

where $k=n 2 \pi / L, n=-N / 2, \ldots, N / 2$. The position of the peaks in Fig. 3 can be explained with the aid of the Fourier transform of the linear part of Eq. (1) with respect to $x$,

$$
\partial_{t} \hat{u}(k, t)=\left[\nu-\left(1-k^{2}\right)^{2}\right] \hat{u}(k, t) .
$$

Equation (3) exhibits a range of linearly unstable wave numbers for $\sqrt{1-\sqrt{\nu}}<k<\sqrt{1+\sqrt{\nu}}$, with $k_{c}=1$ corresponding to the wave number of the most rapidly growing linear mode. Figure 3 shows the time-averaged power spectra at four values of $\nu$. All of them reveal a high peak close to $k_{c}$. Naturally, the peak is not exactly at $k_{c}$ due to nonlinear effects. In Fig. 3(a), the spectra at $\nu=0.625$ (periodic attractor, dotted line) and $\nu=0.632$ (quasiperiodic attractor, solid line) reflect the similarity between the ordered spatial patterns in these regimes. Figure 3(b) displays the difference between the spectra at $\nu=0.635$ (TC attractor, light line) and $\nu=0.637$ (STC attractor, dark line). At $\nu=0.637$, the main peak is much lower and broader, indicating that the spectral energy has spread toward modes in nearby wave numbers. The energy spreading reflects an increase in spatial disorder, which can be quantified by the spectral entropy [31-33]

$$
S(t)=-\sum_{k=1}^{N} p_{k, t} \ln p_{k, t},
$$

where $p_{k, t}$ is the relative weight of mode $k$,
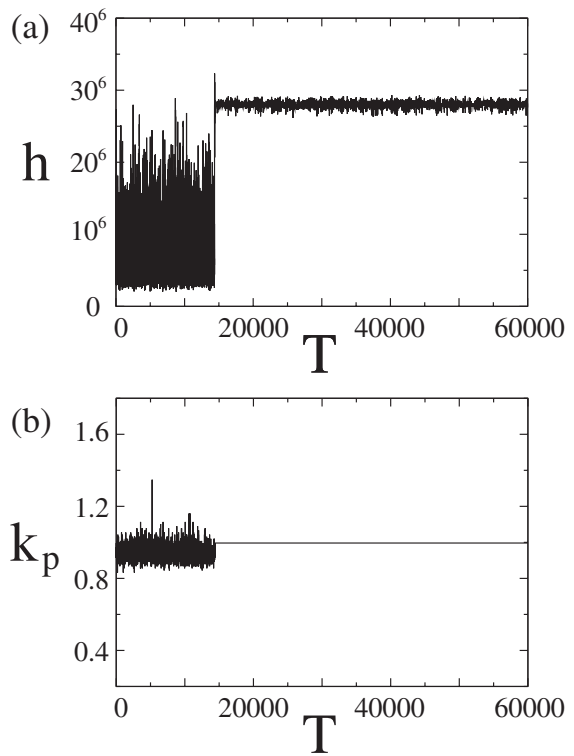

FIG. 4. (a) Time series for the maximum peak height $h$ of the power spectrum as a function of the Poincaré cycles $T$ at temporal chaos regime $(\nu=0.635)$, showing transient spatiotemporal chaos; (b) variation of the wave number $k_{p}$ associated with the maximum peak as a function of $T$.

$$
p_{k, t}=\frac{|\hat{u}(k, t)|^{2}}{\sum_{k}|\hat{u}(k, t)|^{2}},
$$

and the convention $p_{k, t} \ln p_{k, t}=0$ for $p_{k, t}=0$ is used. The normalization in Eq. (5) assures that $p_{k, t} \in[0,1]$ and $\Sigma_{k} p_{k, t}=1$. Thus, if $p_{k, t}=1$, for some $k$, then $S(t)=0$ (perfectly ordered state). The entropy is maximum when $p(k, t)=1 / N, \forall k$ (random state with uniform distribution), in which case it can be shown that $S(t)=\ln N$ [34]. Since $u(x, t)$ is a real function, $|\hat{u}(-k, t)|=|\hat{u}(k, t)|$ and only half of the Fourier modes must be taken into account. Then the maximum entropy is $S(t)=\ln (512) \approx 6.24$. Similar to what happens with the maximum Lyapunov exponent, the time-averaged spectral entropy $\langle S\rangle_{t}$ suddenly increases after the transition from temporal chaos to the spatiotemporal chaos regime. For $\nu=0.635$, $\langle S\rangle_{t} \approx 2.25$, and for $\nu=0.637,\langle S\rangle_{t} \approx 4.36$ [see Fig. 2(b)].

\section{TRANSIENT AND INTERMITTENT SPATIOTEMPORAL CHAOS}

In order to study intermittency in the STC regime, it is crucial to understand the nature of transient spatiotemporal chaos. For $\nu<0.636$, prior to the STC regime, the system displays long periods of STC behavior before converging to a spatially regular attractor. Figure 4(a) illustrates this phenomenon at $\nu=0.635$. The time series for the peak height $h$ of the main peak of the power spectrum is given in terms of the Poincare cycles $T$, i.e., the number of crossings of the flow with the Poincaré section. The time series exhibits high variability and low mean amplitude up to $T \approx 15000$ $(t \approx 250000)$, after which $h$ exhibits low variability and high 

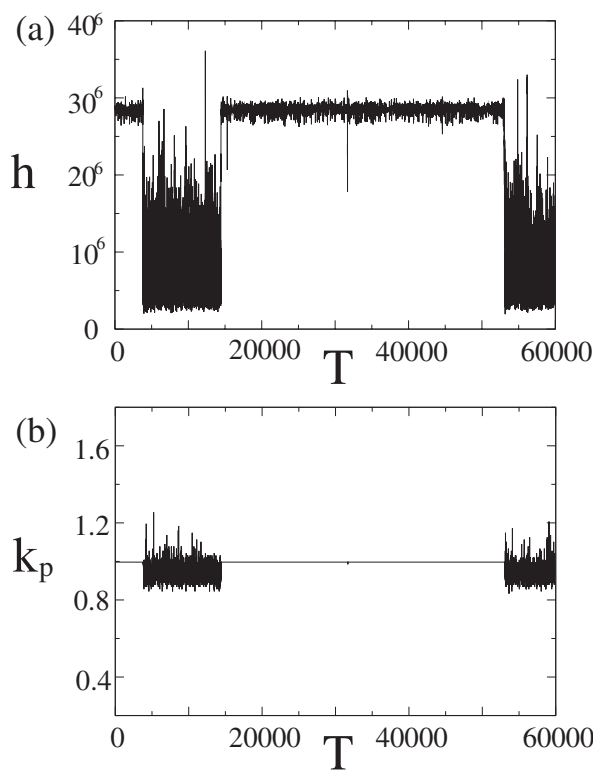

FIG. 5. Intermittent spatiotemporal chaos observed at $\nu=0.636$. (a) Variation of the maximum peak height $h$ of the power spectrum as a function of the Poincaré cycles $T$; (b) variation of the wave number $k_{p}$ associated with the maximum peak as a function of $T$.

mean amplitude. The first part of the time series corresponds to a very long transient spatiotemporal chaos, which is a feature typically shown by random initial conditions. It remains to be verified whether or not this is a signature of supertransient chaos (when the average lifetimes of chaotic transients depend exponentially on the system size $[3,35]$ ) in the damped Kuramoto-Sivashinsky equation. Figure 4(b) shows the corresponding peak position $k_{p}$, i.e., the wave number corresponding to the maximum peak of the power spectrum at a given time. As can be seen, $k_{p}$ is able to clearly distinguish the TC and STC behaviors. During the STC transient, $k_{p}$ varies erratically, in accordance with the complex spatiotemporal structures, where no single characteristic wave number can be identified. Once the TC regime is reached, $k_{p}$ becomes constant, $k_{p}=2 \pi 85 / L$, and refers to the characteristic wave number of the spatial pattern seen in Fig. 1(c).

Right after the transition to STC at $\nu \approx 0.636$, one finds intermittency characterized by "random" switching between periods of TC (lamellar) and STC (bursty) dynamics. Figure 5(a) displays an interval of intermittent time series at $\nu=0.636$. Figure 5(b) shows the corresponding peak position $k_{p}$. During the TC periods, $k_{p}=2 \pi 85 / L$, just as in the TC regime of Fig. 4(b). During the STC periods, $k_{p}$ varies erratically. Note the similarity between the bursty periods in Fig. 5(a) and the transient in Fig. 4, as well as between the lamellar periods in Fig. 5(a) and the final regime in Fig. 4. The STC transient in Fig. 4 corresponds to the dynamics in the neighborhood of a spatiotemporally chaotic saddle (STC saddle). The STC saddle can be found with the sprinkler method $[1,2]$. In the sprinkler method, the chaotic saddle is approximated by points from trajectories that follow long transients before escaping from a predefined restraining region of the phase space. To find the STC saddle, a large set
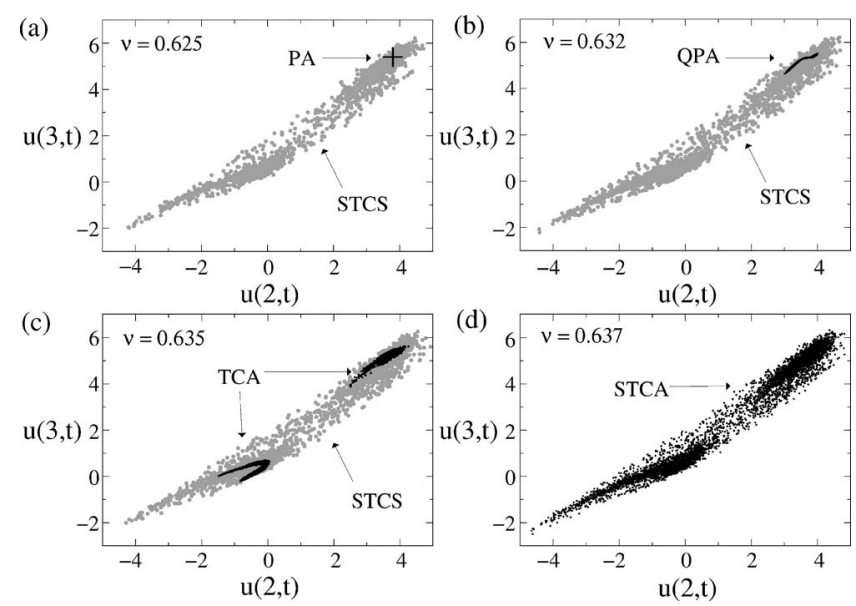

FIG. 6. Two-dimensional projections of the Poincare points of (a) the periodic attractor (PA, cross) and the spatiotemporally chaotic saddle (STCS, gray) at $\nu=0.625$; (b) the quasiperiodic attractor (QPA, black) and STCS (gray) at $\nu=0.632$; (c) the temporally chaotic attractor (TCA, black) and STCS (gray) at $\nu=0.635$; (d) spatiotemporally chaotic attractor (STCA) at $\nu=0.637$.

of initial conditions is iterated by the Poincaré map and those trajectories for which $k_{p} \neq 2 \pi 85 / L$ for 100 consecutive iterations are considered to be in the vicinity of the STC saddle. For each of those trajectories, the first 30 and last 30 iterations are discarded and only 40 points are plotted. This choice of restraining "region" is due to the fact that when the system converges to the TC regime $k_{p}=2 \pi 85 / L$, as mentioned before.

Figure 6 depicts the two-dimensional Poincaré maps $(u(2, t), u(3, t))$ of the attractors and chaotic saddles of the KS equation for four different values of the control parameter $\nu$ (the same values used in Fig. 1). Since the KS equation exhibits multistability, the initial conditions were chosen such that Fig. 6 shows the evolution of a single attractor. In Figs. 6(a)-6(c), the attractor evolves from periodic at $\nu=0.625$ (cross, PA) to quasiperiodic at $\nu=0.632$ (black line, QPA), then to temporally chaotic at $\nu=0.635$ (black points, TCA). The gray points surrounding the attractors represent the STC saddle, responsible for transient STC. At $\nu=0.637$, Fig. 6(d) indicates that the attractor is suddenly enlarged after the transition to STC. It also shows that the STC saddle becomes part of the STC attractor. In fact, by applying the sprinkler method it is possible to find the STC saddle as a subset of the attractor (gray points in Fig. 7). Moreover, by looking for trajectories where $k_{p}=2 \pi 85 / L$ for more than 100 consecutive iterations, one finds, embedded in the STC attractor, a temporally chaotic saddle (TC saddle), which is plotted as black points in Fig. 7. Note that the TC saddle occupies the region previously held by the TC attractor. Since chaotic saddles are always responsible for transient chaos, trajectories on the STC attractor can exhibit transient spatiotemporal chaos whenever they are in the vicinity of the STC saddle, or transient temporal chaos whenever they are in the vicinity of the TC saddle. The recurrence of visits of a trajectory to the vicinities of both chaotic saddles generates the intermittency observed in Fig. 5. We emphasize that, due 


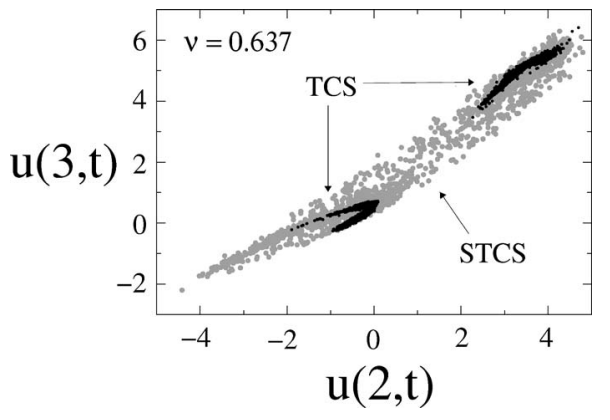

FIG. 7. The temporally chaotic saddle (TCS, black) and spatiotemporally chaotic saddle (STCS, gray) at $\nu=0.637$.

to the long STC transients found close to the TCA-STCA transition, it is very difficult to determine the precise transition point. We have considered $\nu=0.636$ as the transition point based on simulations with time as large as $t=10^{6}$.

Figure 7 reveals that the spatiotemporally chaotic saddle is robust to small changes in the control parameter $\nu$. Even after abrupt changes in the structure of attractors, the STC saddle is only slightly altered. After the transition to STC attractor at $\nu \approx 0.636$, the mean duration of lamellar periods in the intermittent time series drops quickly as $\nu$ increases. Consequently, the bursty phases, ruled by the STC saddle, dominate the dynamics on the STC attractor. Hence, the STC saddle captures the essence of spatiotemporal chaos in the Kuramoto-Sivashinsky equation. In quantitative terms, let us compare the values of the two indicators of temporal and spatial disorder previously mentioned in this work, the maximum Lyapunov exponent $\lambda_{\max }$ and the time-averaged spectral entropy $\langle S\rangle_{t}$, respectively. Figure 2(a) shows the convergence of $\lambda_{\max }$ as a function of time for QPA, TCA, and STCA (black lines) as well as STCS (gray line). Evidently, the Lyapunov exponent of STCS at $\nu=0.632$, in the QP regime, is almost the same as the exponent for STCA at $\nu$ $=0.637$. In Fig. 2(b), $\langle S\rangle_{t}$ is plotted as a function of $\nu$ for both attracting (triangles, A) and nonattracting (squares, STCS) sets. It is clear that the spatial disorder of STCS is the same as that of STCA.

The value of $\langle S\rangle_{t}$ for the attractor in Fig. 2(b) apparently grows linearly between $r=0.635$ and 0.637 . In this range the intermittent lamellar periods due to the TC saddle can be observed in the time series. As $r$ is increased, the average duration of lamellar periods decreases and the STC saddle begins to control most of the dynamics on the attractor. The spectral entropy $\langle S\rangle_{t}$ increases until it saturates at $r=0.637$, when long lamellar periods can barely be observed in time series. The decrease of the average duration of lamellar periods $\tau$ as a function of the distance between $\nu$ and $\nu_{c}$ $=0.636$ follows a power law shown in Fig. 8. The dots represent values computed from long time series and the straight line is a linear fit with slope $\gamma \approx-1.1$.

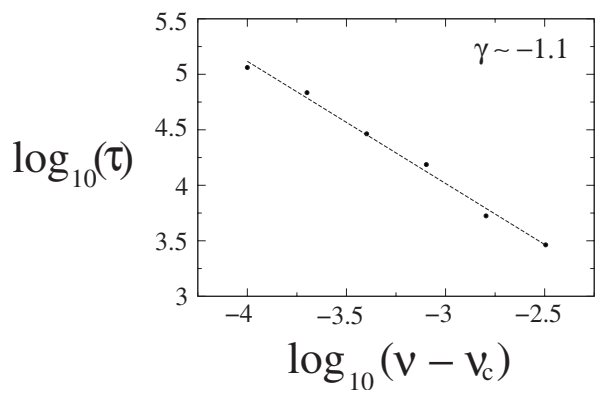

FIG. 8. Decrease of the average duration of lamellar periods $\tau$ as a function of the distance between $\nu$ and $\nu_{c}=0.636$ in log-log scale. The straight line is a linear fit with slope $\gamma \approx-1.1$.

\section{CONCLUSIONS}

The description of transient chaos and TC-STC intermittency in terms of temporally and spatiotemporally chaotic saddles was established for the damped KuramotoSivashinsky equation. The scenario described above suggests that a crisis $[9,26,36-38]$ is responsible for the transition to STC in the damped KS equation. At the critical value of the control parameter, a spatiotemporally chaotic saddle collides with a temporally chaotic attractor and both chaotic sets are merged into a wide spatiotemporally chaotic attractor. The precrisis temporally chaotic attractor loses asymptotic stability, being converted into a temporally chaotic saddle embedded in the spatiotemporally chaotic attractor. The postcrisis enlarged attractor exhibits TC-STC intermittency. This mechanism for intermittency is akin to the coupling between band and surrounding chaotic saddles described in Refs. $[7,39,40]$, which generates crisis-induced intermittency in low-dimensional dynamical systems. Although similar results have been reported for the Kuramoto-Sivashinsky equation in previous works for the temporal chaos regime in small systems $(L=2 \pi)[4,14,15]$, here we report this scenario for a transition to spatiotemporal chaos in a large $(L=536)$ high-dimensional KS system. Since chaotic saddles can be studied in laboratory experiments $[41,42]$, we believe our results can help to improve the understanding of other extended dissipative systems that exhibit crisislike transitions to spatiotemporal chaos, such as the pipe flow experiment [43] and nonlinear optical systems [44].

\section{ACKNOWLEDGMENTS}

This work is supported by CAPES, CNPq, and FAPESP. We thank K. R. Elder for providing the code for solving the KS equation. 
[1] H. Kantz and P. Grassberger, Physica D 17, 75 (1985).

[2] G.-H. Hsu, E. Ott, and C. Grebogi, Phys. Lett. A 127, 199 (1988).

[3] R. Braun and F. Feudel, Phys. Rev. E 53, 6562 (1996).

[4] E. L. Rempel, A. C.-L. Chian, E. E. Macau, and R. R. Rosa, Chaos 14, 545 (2004).

[5] A. Péntek, Z. Toroczkai, T. Tél, C. Grebogi, and J. A. Yorke, Phys. Rev. E 51, 4076 (1995).

[6] E. L. Rempel, W. M. Santana, and A. C.-L. Chian, Phys. Plasmas 13, 032308 (2006).

[7] K. G. Szabó, Y.-C. Lai, T. Tél, and C. Grebogi, Phys. Rev. E 61, 5019 (2000).

[8] Y. Pomeau and P. Manneville, Commun. Math. Phys. 74, 189 (1980).

[9] C. Grebogi, E. Ott, F. Romeiras, and J. A. Yorke, Phys. Rev. A 36, 5365 (1987).

[10] H. Chaté and P. Manneville, Phys. Rev. Lett. 58, 112 (1987).

[11] M. M. Degen, I. Mutabazi, and C. D. Andereck, Phys. Rev. E 53, 3495 (1996).

[12] U. Frisch, Turbulence: The Legacy of A. N. Kolmogorov (Cambridge University Press, Cambridge, U.K., 1996).

[13] Y. Li and C. Meneveau, Phys. Rev. Lett. 95, 164502 (2005).

[14] E. L. Rempel and A. C.-L. Chian, Phys. Lett. A 319, 104 (2003).

[15] E. L. Rempel and Abraham C.-L. Chian, Phys. Rev. E 71, 016203 (2005).

[16] E. L. Rempel and Abraham C.-L. Chian, Phys. Rev. Lett. 98, 014101 (2007).

[17] Y. Kuramoto and T. Tsuzuki, Prog. Theor. Phys. 55, 356 (1976).

[18] G. I. Sivashinsky, Acta Astronaut. 4, 1177 (1977).

[19] R. E. LaQuey et al., Phys. Rev. Lett. 34, 391 (1975).

[20] B. I. Cohen et al., Nucl. Fusion 16, 971 (1976).

[21] K. R. Elder, J. D. Gunton, and N. Goldenfeld, Phys. Rev. E 56, 1631 (1997).

[22] J. M. Hyman and B. Nicolaenko, Physica D 18, 113 (1986).
[23] D. Armbruster, J. Guckenheimer, and P. Holmes, SIAM J. Appl. Math. 49, 676 (1989).

[24] I. G. Kevrekidis, B. Nicolaenko, and J. C. Scovel, SIAM J. Appl. Math. 50, 760 (1990).

[25] F. Christiansen, P. Cvitanović, and V. Putkaradze, Nonlinearity 10, 55 (1997).

[26] A. C.-L. Chian, E. L. Rempel, E. E. Macau, R. R. Rosa, and F. Christiansen, Phys. Rev. E 65, 035203(R) (2002).

[27] R. W. Wittenberg and P. Holmes, Chaos 9, 452 (1999).

[28] M. Hénon, Physica D 5, 412 (1982).

[29] G. Benettin, L. Galvani, A. Giorgilli, and J.-M. Strelcyn, Meccanica 15, 10 (1980).

[30] J. Kurths and A. Brandenburg, Phys. Rev. A 44, R3427 (1991).

[31] G. E. Powell and I. C. Percival, J. Phys. A 12, 2053 (1979).

[32] H. Xi and J. D. Gunton, Phys. Rev. E 52, 4963 (1995).

[33] R. V. Cakmur, D. A. Egolf, B. B. Plapp, and E. Bodenschatz, Phys. Rev. Lett. 79, 1853 (1997).

[34] R. Badii and A. Politi, Complexity: Hierarchical Structures and Scaling in Physics (Cambridge University Press, Cambridge, U.K., 1997).

[35] K. Kaneko, Phys. Lett. A 149, 105 (1990).

[36] C. Grebogi, E. Ott, and J. A. Yorke, Physica D 7, 181 (1983).

[37] K. G. Szabó and T. Tél, Phys. Rev. E 50, 1070 (1994).

[38] F. A. Borotto, A. C.-L. Chian, and E. L. Rempel, Int. J. Bifurcation Chaos Appl. Sci. Eng. 14, 2375 (2004).

[39] K. G. Szabó and T. Tél, Phys. Lett. A 196, 173 (1994).

[40] K. G. Szabó, Y.-C. Lai, T. Tél, and C. Grebogi, Phys. Rev. Lett. 77, 3102 (1996)

[41] I. M. Jánosi, L. Flepp, and T. Tél, Phys. Rev. Lett. 73, 529 (1994).

[42] S. Banerjee, IEEE Trans. Circ. Syst. 44, 847 (1997).

[43] J. Peixinho and T. Mullin, Phys. Rev. Lett. 96, 094501 (2006).

[44] M. Sauer and F. Kaiser, Int. J. Bifurcation Chaos Appl. Sci. Eng. 6, 1481 (1996). 